\title{
Cystine Urolithiasis
}

National Cancer Institute

\section{Source}

National Cancer Institute. Cystine Urolithiasis. NCI Thesaurus. Code C123244.

Urolithiasis in which the composition of the stones is predominantly cystine. 